\title{
Studies on Whole Cell Fluorescence-Based Screening for Epoxide Hydrolases and Baeyer- Villiger Monoxygenases
}

\author{
Beatriz Bicalho $^{a}$, Lu S. Chen ${ }^{a}$, Johann Grognux ${ }^{b}$,Jean-Louis Reymond ${ }^{b}$ and Anita J. Marsaioli ${ }^{*, a}$ \\ ${ }^{a}$ Instituto de Química, Universidade Estadual de Campinas, CP 6154, 13083-970 Campinas - SP, Brazil \\ ${ }^{b}$ University of Berne, Department of Chemistry and Biochemistry, Freiestrasse 3, CH-3012, Berne, Switzerland
}

\begin{abstract}
Reações de biocatálise foram realizadas em microplacas $(200 \mu \mathrm{L})$ visando a utilização de substratos fluorogênicos (100 $\left.\mu \mathrm{mol} \mathrm{L}^{-1}\right)$ para prospecção rápida de epóxido hidrolases (EHs) e Baeyer-Villiger monoxigenases (BVMOs) em microrganismos (células inteiras). Um protocolo final foi alcançado para EHs, com a detecção de 3 novas fontes enzimáticas (Agrobacterium tumefaciens, Pichia stipitis, Trichosporom cutaneum). O ensaio fluorogênico para BVMO não ocorreu como esperado. A abordagem de algumas variáveis envolvidas (aeração; $\mathrm{pH}$ ) proporcionou a detecção inédita da atividade enzimática de BVM em T. cutaneum.
\end{abstract}

Biocatalysis reactions were performed on microtiter plates $(200 \mu \mathrm{L})$ aiming at the utilization of fluorogenic substrates $\left(100 \mu \mathrm{mol} \mathrm{L}^{-1}\right)$ for rapid whole cell screening for epoxide hydrolases (EHs) and Baeyer-Villiger monoxygenases (BVMOs). A final protocol was achieved for EHs, with 3 new enzymatic sources being detected (Agrobacterium tumefaciens, Pichia stipitis, Trichosporom cutaneum). The fluorogenic assay for BVMO did not work as expected. However, an approach to possible variables involved (aeration; $\mathrm{pH}$ ) provided the first detection of a BVMO activity in $T$. cutaneum.

Keywords: microorganisms, Baeyer-Villiger monoxygenase, epoxide hydrolase

\section{Introduction}

A challenge that chemists are presently facing is the development of more efficient and cleaner routes to make old and new products. It is here that catalysts such as enzymes are unique making the best of raw material saving energy and replacing toxic reagents. Notwithstanding these qualities enzymes can display high stereoselectivities and that is why biotechnological processes are developing rapidly in industrial organic synthesis. It is also true that efficient biocatalysts are nowadays limited because industrial substrates are usually artificial and suitable enzymes are often unknown. Therefore, special biocatalysts must be found by screening microorganisms or any other biological source rich in enzymes and this have been a reason for several achievements in catalysis assays applied to screening enzyme activity in high throughput format with liquid chromatography-mass spectrometry (LC-MS),

* e-mail: anita@iqm.unicamp.br capillary electrophoresis, and assays based on fluorogenic or chromogenic substrates. ${ }^{1-5}$

The presence of useful biocatalysts in microorganisms of two Brazilian Culture Collections (CCT - Coleção de Culturas Tropical, Fundação Tropical de Pesquisas André Tosello; CBMAI - Coleção Brasileira de Microrganismos de Ambiente e Indústria, Centro Pluridisciplinar de Pesquisas Químicas, Biológicas e Agrícolas, UNICAMP) have been investigated. ${ }^{6-8}$ However, the time-consuming procedures typical of classical methodologies for screening whole cell enzymatic activity have been a critical drawback. Although high throughput screening (HTS) assays are being rapidly innovated, ${ }^{2,9-11}$ whole cell HTS assays have been almost neglected, excepting those based on fluorogenic substrates which have been applied to metagenomic libraries (in function-driven analysis) but seldom to natural culture collections. In order to address this problem, this paper reports the application of a 96well microplate fluorogenic assay, originally designed for purified hydrolytic enzymes, ${ }^{12,13}$ to screen microbial whole cells for epoxide hydrolases (EHs) and Baeyer-Villiger monooxygenases (BVMOs). 


\section{Results and Discussion}

The experimental approach herein described was adapted from the high throughput screening assay methodology introduced by Reymond and Klein ${ }^{12}$ (Scheme 1) and by Furstoss and co-workers ${ }^{14}$ (Scheme 2). Epoxides, probe-substrates for $\mathrm{EH}$, were known to endure the assay conditions. ${ }^{4,12}$ The same was not true for ketone $\mathbf{9}$, therefore its stability in borate buffer $\mathrm{pH} 8.8$ had to be evaluated in the presence and in the absence of the assay components (Scheme 2), namely horse liver alcohol dehydrogenase $(\mathrm{HLADH}), \mathrm{NAD}^{+}$and BSA. No time-dependent increase in fluorescence was observed for any combination of reagents within $48 \mathrm{~h}$, discarding the formation of $\mathbf{1 1}$ and release of $\mathbf{7}$ in the absence of a specific Baeyer-Villiger monooxygenase.
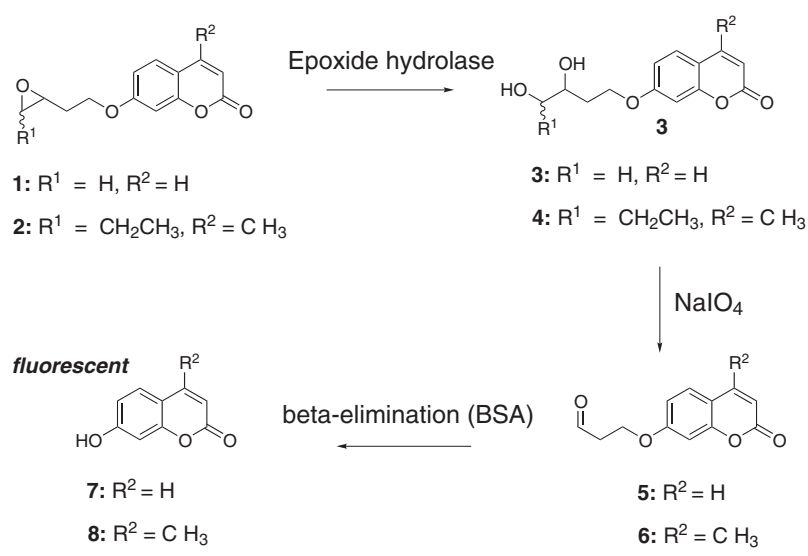

Scheme 1. Fluorogenic epoxide hydrolase assay with epoxides $\mathbf{1}$ and 2.

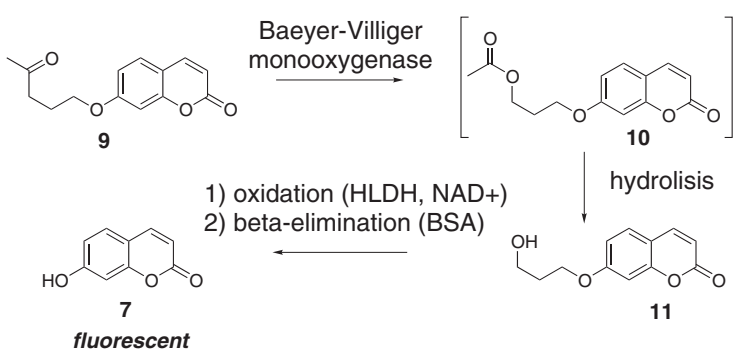

Scheme 2. Fluorogenic Baeyer-Villiger monooxygenase assay with ketone 9.

In order to validate the oxidation of $\mathbf{1 1}$ by HLADH/ $\mathrm{NAD}^{+}$and subsequent $\beta$-elimination step (Scheme 2 ), the fluorescence of a borate buffer solution $(\mathrm{pH} 8.8)$ of $\mathbf{1 1}$ and BSA with or without HLADH/NAD ${ }^{+}$was evaluated. A significant time-dependent increase in fluorescence intensity was only observed in the presence of HLADH/ $\mathrm{NAD}^{+}$, thus indicating the release of $\mathbf{7}$ due to the oxidation of 11.

To adjust the enzymatic assay to the cell format, fixed amounts of diol 3, metaperiodate $\left(\mathrm{NaIO}_{4}\right)$, and BSA (see
Scheme 1) were set to react in the presence of increasing concentrations of Rhodotorula glutinis CCT 2182. As shown in Figure 1, the time-dependent increase in fluorescence intensity due to $\mathbf{7}$ released as final product was inversely proportional to cell concentration. The presence of $0.1 \mathrm{mg} \mathrm{mL}^{-1}$ cells in the solution was not detrimental to the lecture, as estimated from the control reaction. Thus, this limit was established as the maximum cell concentration to run the screening assays. The microorganisms used (Table 1) were cultivated for 3 days on appropriate agar media, at room temperature. The colonies were collected and suspended in borate buffer $\mathrm{pH} 8.8$ just prior to the reactions, which were performed without cell' substrate pre-incubation.

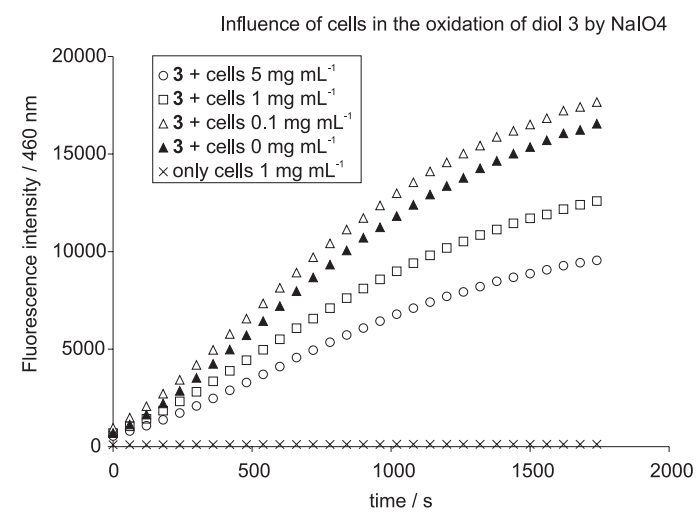

Figure 1. Fluorescence $(460 \mathrm{~nm})$ in borate buffer $\left(20 \mathrm{mmol} \mathrm{L}^{-1}, \mathrm{pH}\right.$ $8.8,30{ }^{\circ} \mathrm{C}$ ) due to the conversion $\mathbf{3} \rightarrow \mathbf{7}$ by $\mathrm{NaIO} / \mathrm{BSA}$ in the presence of increasing concentrations of $R$. glutinis CCT 2182. Diol $3\left(100 \mu \mathrm{mol} \mathrm{L}^{-1}\right), \mathrm{NaIO}_{4}\left(1 \mathrm{mmol} \mathrm{L}^{-1}\right)$, BSA $\left(2 \mathrm{mg} \mathrm{mL}^{-1}\right)$, cells (dry weigh, $0-5 \mathrm{mg} \mathrm{mL}^{-1}$ ).

Table 1. Microorganisms screened for EH and BVMO activities

\begin{tabular}{|c|c|}
\hline Microorganisms & Original environment \\
\hline Geotrichum candidum CCT 1205 & $\begin{array}{l}\text { Metal contaminated } \\
\text { industrial fluid. } \\
\text { Preston. United Kindon }\end{array}$ \\
\hline Pichia kluyeri CCТ 3365 & $\begin{array}{l}\text { Fruit of Peurouma } \\
\text { guianensis. Altlantic } \\
\text { Forest (SP). Brazil }\end{array}$ \\
\hline Pichia stipitis CCT 2617 & Insect larvae. France \\
\hline Rhodotorula glutinis CCT 2182 & Leaves of Guará tree \\
\hline Rhodotorula minuta CCT 1751 & $\begin{array}{l}\text { Cerrado soil. } \\
\text { Corumbataí (SP). } \\
\text { Brazil }\end{array}$ \\
\hline Trichosporon cutaneum CCT 1903 & $\begin{array}{l}\text { Petroleum refinery } \\
\text { sulfate. Japan }\end{array}$ \\
\hline Agrobacterium tumefaciens CCT 6515 & Not specified \\
\hline Citrobacter amalonaticus CCT 4059 & $\begin{array}{l}\text { Poultry } \\
\text { slaughtherhouse. Brazil }\end{array}$ \\
\hline Pseudomonas aeruginosa CCT 1987 & Outer ear infection \\
\hline Pseudomonas aeruginosa CCT 2738 & $\begin{array}{l}\text { Animal room water } \\
\text { bottle }\end{array}$ \\
\hline Serratia rubidea CCT 5732 & Coconut fruit. Brazil \\
\hline
\end{tabular}

CCT: Coleção de Culturas Tropical. 


\section{Screening for $\mathrm{EH}$}

R. glutinis was included among the 11 microrganisms as a positive control due to its known EH activity. ${ }^{6,15,16}$ The reactions with $\mathbf{1}$ and $\mathbf{2}$ (Scheme 1) were monitored for $10 \mathrm{~h}$. The time-dependent increase in fluorescence intensity revealed three different $\mathrm{EH}$ biocatalysts. The $\mathrm{EH}$ activity revealed by $T$. cutaneum CCT 1903 was selective for 1 while that of A. tumefaciens CCT 6515 or P. stipitis CCT 2617 was selective for 2 . On the other hand, the EH activity of $R$. glutinis CCT 2182 did not discriminate between 1 and 2 (Figures 2 and 3). T. cutaneum, A. tumefaciens or $P$. stipitis have never been mentioned before as EH sources, and we are now investigating the site and stereoselectivity of the detected biocatalysts.

\section{Screening for BVMO}

Following the same strategy described for the $\mathrm{EH}$, fungus G. candidum CCT 1205 was included among the investigated microorganisms, as it was known to convert

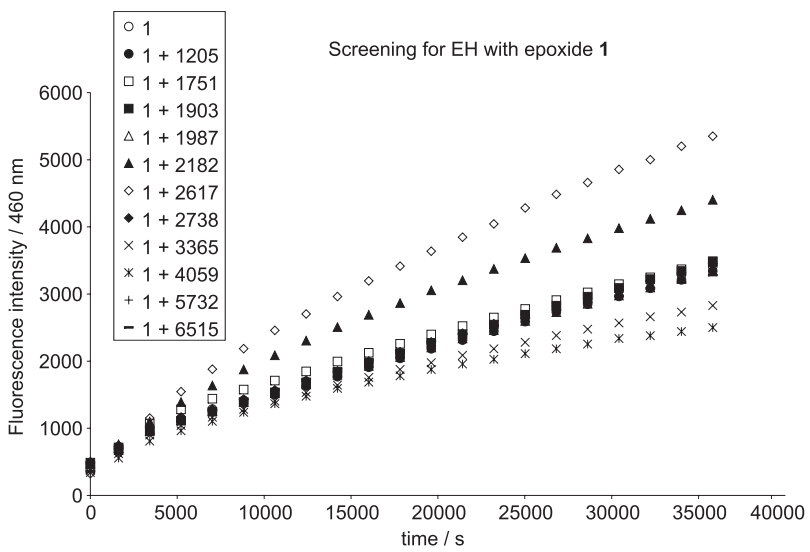

Figure 2. Development of fluorescence intensity $(460 \mathrm{~nm})$ in borate buffer $\left(20 \mathrm{mmol} \mathrm{L}^{-1}, \mathrm{pH} 8.8,30^{\circ} \mathrm{C}\right)$ due to reactions of epoxide $1\left(100 \mu \mathrm{mol} \mathrm{L}^{-1}\right)$ with microbial cells $\left(0.1 \mathrm{mg} \mathrm{mL}^{-1}\right)$ in the presence of $\mathrm{NaIO}_{4}\left(1 \mathrm{mmol} \mathrm{L}^{-1}\right)$ and BSA $\left(2 \mathrm{mg} \mathrm{mL}^{-1}\right)$. To correlate legend numbers to microorganisms see Table 1 . methyl-cyclohexanones into methyl- $\varepsilon$-caprolactones. ${ }^{17}$ However, monitoring BVMO activity applying the fluorogenic HTS protocol (Scheme 2) was not as straightforward as expected. No significant time-dependent increase in fluorescence was detected, and even after $48 \mathrm{~h}$ of incubation only a very discrete fluorescence increment was registered for the reaction with T. cutaneum CCT 1903. Assigning these results to a putative low oxygenation level of the microtiter plate, traditional resting cell biocatalysis of ketone $\mathbf{9}$ into ester $\mathbf{1 0}$ or alcohol $\mathbf{1 1}$ (Scheme 2) by G. candidum CCT 1205 or T. cutaneum CCT 1903 was monitored by HPLC. In these reactions the release of 7 was not expected as no $\mathrm{HLADH} / \mathrm{NAD}^{+}$and BSA were added to the medium. However, in accordance with the previous results, no time-dependent significant increase in compounds $\mathbf{1 0}$ or $\mathbf{1 1}$ was detected (Table 2).

As the BVMO of G. candidum CCT 1205 was previously detected in aqueous phosphate $(\mathrm{pH} 6.5),{ }^{17}$ the buffer influence on the BVMO activity was investigated by performing the reaction in this condition. However, the conversion of 9 into $\mathbf{1 1}$ by G. candidium CCT 1205 in

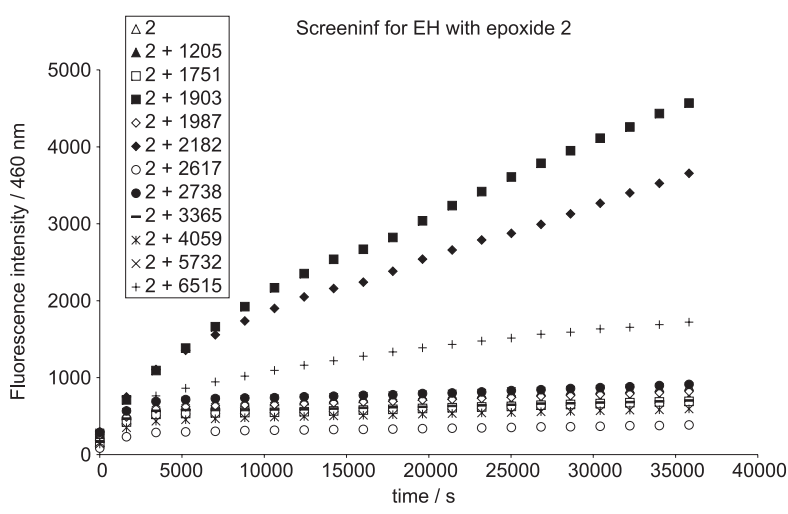

Figure 3. Fluorescence $(460 \mathrm{~nm})$ in borate buffer $\left(20 \mathrm{mmol} \mathrm{L}^{-1}, \mathrm{pH}\right.$ $\left.8.8,30^{\circ} \mathrm{C}\right)$ due to reactions of epoxide $2\left(100 \mu \mathrm{mol} \mathrm{L}^{-1}\right)$ with microbial cells $\left(0.1 \mathrm{mg} \mathrm{mL}^{-1}\right)$ in the presence of $\mathrm{NaIO}_{4}\left(1 \mathrm{mmol} \mathrm{L}^{-1}\right)$ and BSA $\left(2 \mathrm{mg} \mathrm{mL}^{-1}\right)$. To correlate legend numbers to microorganisms see Table 1 .

Table 2. Biocatalysis of 9 in borate buffer $\mathrm{pH} 8.8$ monitored by HPLC

\begin{tabular}{|c|c|c|c|c|c|c|c|c|c|c|c|c|}
\hline \multirow[b]{2}{*}{$\mathrm{t}^{\mathrm{c}}$} & \multicolumn{4}{|c|}{$\begin{array}{c}\text { G. candidum CCT } 1205 \\
\text { A\% }\end{array}$} & \multicolumn{4}{|c|}{$\begin{array}{c}\text { T. cutaneum CCT } 1903 \\
\text { A\% }\end{array}$} & \multicolumn{4}{|c|}{$\frac{\mathrm{C}^{\mathrm{a}}}{\mathrm{A} \%}$} \\
\hline & 7 & 9 & 10 & 11 & 7 & 9 & 10 & 11 & 7 & 9 & 10 & 11 \\
\hline 24 & 0.1 & 98.1 & - & 0,4 & 0,6 & 96,9 & - & 1,1 & nd & nd & nd & nd \\
\hline 48 & $\mathrm{nd}$ & $\mathrm{nd}$ & $\mathrm{nd}$ & $\mathrm{nd}$ & $\mathrm{nd}$ & $\mathrm{nd}$ & $\mathrm{nd}$ & $\mathrm{nd}$ & nd & nd & nd & $\mathrm{nd}$ \\
\hline 72 & 0.5 & 98.3 & - & 0,1 & 0,7 & 96,5 & - & 2,0 & $\mathrm{nd}$ & $\mathrm{nd}$ & $\mathrm{nd}$ & $\mathrm{nd}$ \\
\hline 96 & 0.6 & 98.2 & - & 0,1 & 0,7 & 96,1 & - & 2,2 & $\mathrm{nd}$ & nd & $\mathrm{nd}$ & $\mathrm{nd}$ \\
\hline 144 & $\mathrm{nd}$ & nd & nd & $\mathrm{nd}$ & 0,6 & 95,2 & - & 2,2 & nd & nd & nd & nd \\
\hline
\end{tabular}

${ }^{a}$ Control (reactional mixture without microbial cells). ${ }^{b}$ Percentual chromatographic area. ${ }^{\mathrm{c}}$ Reaction time (hours). nd: not detemined. -: not detected. 
Table 3. Biocatalysis of $\mathbf{9}$ in phosphate buffer $\mathrm{pH} 6.5$ monitored by HPLC

\begin{tabular}{|c|c|c|c|c|c|c|c|c|c|c|c|c|}
\hline \multirow[b]{2}{*}{$t^{c}$} & \multicolumn{4}{|c|}{$\begin{array}{c}\text { G. candidum CCT } 1205 \\
\text { A\% }\end{array}$} & \multicolumn{4}{|c|}{$\begin{array}{c}\text { T. cutaneum CCT } 1903 \\
\text { A\% }\end{array}$} & \multicolumn{4}{|c|}{$\frac{\mathrm{C}^{\mathrm{a}}}{\mathrm{A} \%}$} \\
\hline & 7 & 9 & 10 & 11 & 7 & 9 & 10 & 11 & 7 & 9 & 10 & 11 \\
\hline 24 & 0.6 & 97.6 & - & 1.3 & 0.6 & 86.5 & - & 12.3 & 0.6 & 99.2 & - & - \\
\hline 48 & 0.6 & 96.1 & - & 2.8 & 0.9 & 70.4 & - & 27.7 & 0.6 & 99.2 & - & - \\
\hline 72 & $\mathrm{nd}$ & nd & $\mathrm{nd}$ & $\mathrm{nd}$ & 1.8 & 52.4 & - & 45.0 & 0.6 & 99.2 & - & - \\
\hline
\end{tabular}

${ }^{a}$ Control (reactional mixture without microbial cells). ${ }^{b}$ Percentual chromatographic area. ${ }^{c}$ Reaction time (hours). nd: not detemined. -: not detected.

aqueous phosphate $(\mathrm{pH} 6.5)$ did not even reach $3 \%$ within $48 \mathrm{~h}$. On the other, the lower $\mathrm{pH}$ was found essential to activate the BVMO of T. cutaneum CCT 1903, as almost $30 \%$ of the starting 9 was converted into 11 within $48 \mathrm{~h}$. In both cases the intermediate $\mathbf{1 0}$ was never detected, probably due to the action of hydrolytic enzymes such as lipases or esterases, as previously mentioned. ${ }^{14}$

As reviewed recently, BVMOs are classified according to substrate specificity, ${ }^{18}$ therefore BVMOs of G. candidum CCT 1205 and T. cutaneum CCT 1903 belong to different groups. Therefore, probe-substrates for enzyme activity must be carefully selected as "you get what you screen for". Taking this in consideration we have tested both microorganisms in whole cell biocatalysis experiments using several ketones confirming the presence of $\mathrm{CHMO}$ in G. candidum and BVMO for special alkanones in $T$. cutaneum, these results will be published elsewhere.

The oxidation of $\mathbf{1 1}$ by HLADH/NAD ${ }^{+}$in phosphate buffer $\mathrm{pH} 6.5$ did not work satisfactorily, preventing the application of the Scheme 2 to screening for BVMO activity.

\section{Experimental}

\section{7-(2'-oxyranyl-ethoxy)-2H-1-benzopyran-2-one, (1)}

A mixture of 7-hydroxy-2H-1-benzopyran-2-one (0.91 g, $5.6 \mathrm{mmol}), \mathrm{NaH}$ (60\% suspension in oil; $0.61 \mathrm{~g}, 15 \mathrm{mmol}$ ) and 3-butenyl $p$-toluenosulfonate $(1.14 \mathrm{~g}, 5 \mathrm{mmol})$ were stirred in DMF $(20 \mathrm{~mL}$ ) for $24 \mathrm{~h}$. The mixture was then diluted with AcOEt and washed successively with $\mathrm{H}_{2} \mathrm{O}$ and $1 \mathrm{~mol} \mathrm{~L}^{-1}$ aqueous $\mathrm{NaOH}$. Evaporation of the organic phase and flash chromatography (FC; hexane/AcOEt gradient) gave the olefinic precursor of 1 , which $(0.10 \mathrm{~g}$, $0.46 \mathrm{mmol})$ was dissolved in $\mathrm{CH}_{2} \mathrm{Cl}_{2}(3 \mathrm{~mL})$ containing MCPBA $(0.10 \mathrm{~g}, 0.55 \mathrm{mmol})$. After $24 \mathrm{~h}$ at ambient temperature, the mixture was diluted with $\mathrm{CH}_{2} \mathrm{Cl}_{2}$ and washed successively with $10 \%$ aqueous $\mathrm{Na}_{2} \mathrm{SO}_{3}$ and 0.1 mol L ${ }^{-1}$ aqueous sodium bicarbonate. Evaporation of the organic phase and FC (hexane/EtOAc gradient) gave $\mathbf{1}$ (0.85 g, $0.37 \mathrm{mmol}, 80 \%$ ) as colorless crystals; mp 67.8 -
69.3을 (EtOAc). lit. $^{12} 60$ - $64{ }^{\circ} \mathrm{C} .{ }^{1} \mathrm{H}$ NMR (300.067 MHz, $\left.\mathrm{CDCl}_{3}, \delta_{\mathrm{TMS}} 0.00 \mathrm{ppm}\right) \delta 7.45\left(1 \mathrm{H}, \mathrm{d},{ }^{3} J 9.2\right), 7.38(1 \mathrm{H}, \mathrm{d}$, ${ }^{3} J$ 8.4), 6.85 (1 H, dd, ${ }^{3} J$ 8.4; 2.5), 6.83 (1 H, d, $\left.{ }^{3} J 2.5\right), 6.26$ (1 H, d, $\left.{ }^{3} J 9.2\right), 4.19$ (2 H, m), 3.17 (1 H, m), $2.86\left(1 \mathrm{H}, \mathrm{t},{ }^{3} J\right.$ 4.7), 2.60 (1 H, dd, $\left.{ }^{3} J 4.7 ; 2.7\right), 2.19$ ( $\left.1 \mathrm{H}, \mathrm{m}\right), 1.95$ (1 H, m); ${ }^{13} \mathrm{C}$ NMR (75.45 MHz, $\left.\mathrm{CDCl}_{3}, \delta_{\mathrm{CDCl}_{3}} 77.0 \mathrm{ppm}\right) \delta 161.7$ (C), $161.0(\mathrm{C}), 155.7(\mathrm{C}), 143.2(\mathrm{CH}), 128.7(\mathrm{CH}), 113.1$ $(\mathrm{CH}), 112.7(\mathrm{CH}), 112.6(\mathrm{C}), 101.4(\mathrm{CH}), 65.3\left(\mathrm{CH}_{2}\right), 49.5$ $(\mathrm{CH}), 47.2\left(\mathrm{CH}_{2}\right), 32.3\left(\mathrm{CH}_{2}\right)$; EIMS , $\mathrm{m} / z, 232\left(\mathrm{M}^{+}, 86 \%\right)$, 162 (42), 134 (100), 89 (29), 71 (34).

A similar procedure starting with cis-3-hexenyl p-toluenosulfonate (1.66 g, $6.5 \mathrm{mmol})$ and 7-hydroxy-4methyl-2H-1-benzopyran-2-one (1.26 g, $7.1 \mathrm{mmol})$ gave a diasteroisomeric mixture of cis/trans (1.5:1.0) 2 as a pale oil (1.26 g, 73\%). ${ }^{1} \mathrm{H}$ NMR (499.883 MHz, $\mathrm{CDCl}_{3}, \delta_{\mathrm{TMS}}$ $0.00 \mathrm{ppm})$, cis-isomer, $\delta 7.50\left(1 \mathrm{H}, \mathrm{d},{ }^{3} J\right.$ 8.7), 6.87 (1 H, dd, ${ }^{3} J$ 8.7; 2.5), 6.83 (1 H, d, $\left.{ }^{3} J 2.5\right), 6.15$ (1 H, s), 4.20 (2 H, m), 3.19 (1H, ddd, ${ }^{3} J$ 7.3 , 4.6, 4.6), 2.99 (1H, ddd, ${ }^{3} J$ 6.4, 6.4, 4.3), 2.15 (1 H, m), 1.96 (1 H, m), 1.09 (3H, t, ${ }^{3}$ J 7.6); transisomer, $\delta 7.50\left(1 \mathrm{H}, \mathrm{d},{ }^{3} J\right.$ 8.7), $6.87\left(1 \mathrm{H}, \mathrm{dd},{ }^{3} J\right.$ 8.7; 2.5), $6.83\left(1 \mathrm{H}, \mathrm{d},{ }^{3} \mathrm{~J} 2.5\right), 6.15(1 \mathrm{H}, \mathrm{s}), 4.16(2 \mathrm{H}, \mathrm{m}), 2.94(1 \mathrm{H}$, ddd, $\left.{ }^{3} J 6.2,4.6,2.1\right), 2.79$ (1H, ddd, $\left.{ }^{3} J 5.5,5.5,2.1\right), 1.61$ (2 $\mathrm{H}, \mathrm{m}), 1.01$ (3H, t, $\left.{ }^{3} \mathrm{~J} 7.6\right) ;{ }^{13} \mathrm{C} \mathrm{NMR}\left(75.45 \mathrm{MHz}, \mathrm{CDCl}_{3}\right.$, $\delta_{\mathrm{CDCl}_{3}} 77.0 \mathrm{ppm}$ ) cis-isomer, $\delta 161.8(\mathrm{C}), 161.2(\mathrm{C}), 155.3$ (C), $152.4(\mathrm{C}), 125.6(\mathrm{CH}), 113.7(\mathrm{C}), 112.4(\mathrm{CH}), 112.0$ $(\mathrm{CH}), 101.7(\mathrm{CH}), 65.8\left(\mathrm{CH}_{2}\right), 58.3(\mathrm{CH}), 54.3(\mathrm{CH}), 27.7$ $\left(\mathrm{CH}_{2}\right), 21.6\left(\mathrm{CH}_{2}\right), 18.6\left(\mathrm{CH}_{3}\right), 10.4\left(\mathrm{CH}_{3}\right)$; trans-isomer, $\delta$ $161.8(\mathrm{C}), 161.2(\mathrm{C}), 155.3(\mathrm{C}), 152.4(\mathrm{C}), 125.6(\mathrm{CH})$ $113.7(\mathrm{C}), 112.4(\mathrm{CH}), 112.0(\mathrm{CH}), 101.7(\mathrm{CH}), 65.2\left(\mathrm{CH}_{2}\right)$, $60.1(\mathrm{CH}), 55.4(\mathrm{CH}), 31.8\left(\mathrm{CH}_{2}\right), 24.9\left(\mathrm{CH}_{2}\right), 18.6\left(\mathrm{CH}_{3}\right)$, $9.8\left(\mathrm{CH}_{3}\right)$; cis-isomer, EIMS: $\mathrm{m} / z 274\left(\mathrm{M}^{+}, 26 \%\right), 256(7)$, 188 (30), 176 (58), 148 (100); trans-isomer, EIMS: $\mathrm{m} / \mathrm{z}$ $274\left(\mathrm{M}^{+}, 100 \%\right), 256$ (12), 188 (18), 176 (44), 148 (46).

7-(4'-oxo-pentyl)-2H-1-benzopyran-2-one, (9)

A mixture of 7-hydroxy-2H-1-benzopyran-2-one (0.57 g, $3.5 \mathrm{mmol}$ ), $\mathrm{NaH}$ ( $60 \%$ suspension in oil; $0.4 \mathrm{~g}, 10 \mathrm{mmol}$ ) and 4-pentenyl p-toluenosulfonate $(0.8 .4 \mathrm{~g}, 3.5 \mathrm{mmol})$ were stirred in DMF (15 mL) for $24 \mathrm{~h}$. The mixture was then diluted with EtOAc and washed successively with $\mathrm{H}_{2} \mathrm{O}$ and 
$1 \mathrm{~mol} \mathrm{~L}^{-1}$ aqueous $\mathrm{NaOH}$. Evaporation of the organic phase and $\mathrm{FC}$ (hexane/AcOEt gradient) gave the olefinic precursor of 9 , which $(0.16 \mathrm{~g}, 0.69 \mathrm{mmol})$ was dissolved in a mixture of $\mathrm{PdCl}_{2}(12.1 \mathrm{mg}, 0.07 \mathrm{mmol})$ and $\mathrm{CuCl}(68.3 \mathrm{mg}, 0.69$ $\mathrm{mmol})$ in DMF- $\mathrm{H}_{2} \mathrm{O}(5 \mathrm{~mL}, 7: 1)$ under $\mathrm{O}_{2}$ atmosphere. After $24 \mathrm{~h}$ at ambient temperature the mixture was diluted with $5 \%$ aqueous $\mathrm{HCl}$ and washed with EtOAc. Evaporation of the organic phase and FC (hexane/AcOEt gradient) gave 9 $(0.11 \mathrm{~g}, 64 \%)$ as colorless crystals; mp $74.4-75.8^{\circ} \mathrm{C}$ (EtOAc). ${ }^{1} \mathrm{H}$ NMR (300.067 MHz, $\left.\mathrm{CDCl}_{3}, \delta_{\mathrm{TMS}} 0.00 \mathrm{ppm}\right) \delta$ 7.62 (d, $1 \mathrm{H},{ }^{3} J$ 9.5), 7.35 (d, $\left.1 \mathrm{H},{ }^{3} J 8.2\right), 6.80$ (dd, $1 \mathrm{H},{ }^{3} J$ 8.2; 2.5), $6.76\left(\mathrm{~d}, 1 \mathrm{H},{ }^{3} J 1.8\right), 6.22\left(\mathrm{~d}, 1 \mathrm{H},{ }^{3} J 9.5\right), 4.02\left(\mathrm{t}, 2 \mathrm{H},{ }^{3} \mathrm{~J}\right.$ 6.0), $2.66\left(\mathrm{t}, 2 \mathrm{H},{ }^{3} \mathrm{~J} 7.1\right), 2.17$ (s, $\left.3 \mathrm{H}\right), 2.08\left(2 \mathrm{H}, \mathrm{qt},{ }^{3} \mathrm{~J} 6.6\right.$ ); ${ }^{13} \mathrm{C} \mathrm{NMR}\left(75.45 \mathrm{MHz}, \mathrm{CDCl}_{3}, \delta_{\mathrm{CDCl}_{3}} 77.0 \mathrm{ppm}\right) \delta 207.5(\mathrm{C})$, $161.8(\mathrm{C}), 160.9(\mathrm{C}), 155.5(\mathrm{C}), 143.2(\mathrm{CH}), 128.6(\mathrm{CH})$, $112.9(\mathrm{CH}), 112.5(\mathrm{CH}), 112.4(\mathrm{C}), 101.3(\mathrm{CH}), 67.4\left(\mathrm{CH}_{2}\right)$, $39.6\left(\mathrm{CH}_{2}\right), 30.1\left(\mathrm{CH}_{2}\right), 23.0\left(\mathrm{CH}_{3}\right)$; EIMS: $\mathrm{m} / z 246\left(\mathrm{M}^{+}\right.$, 5\%), 162 (7), 134 (31), 85 (100), 43 (75).

\section{7-(3'-acetoxy-propyl)-2H-1-benzopyran-2-one, (10)}

Acetyl chloride $(0.69 \mathrm{~g}, 8.5 \mathrm{mmol})$ was added to 3propanol $p$-toluenosulfonate $(1.15 \mathrm{~g}, 5 \mathrm{mmol})$ and pyridine (1.5 mL, $17.5 \mathrm{mmol})$ in $\mathrm{CH}_{2} \mathrm{Cl}_{2}(6 \mathrm{~mL})$. After $30 \mathrm{~min}$ the mixture was diluted with EtOAc $(50 \mathrm{~mL})$ and washed successively with $0.1 \mathrm{~mol} \mathrm{~L}^{-1}$ aqueous $\mathrm{NaHCO}_{3}, 10 \%$ aqueous $\mathrm{CuSO}_{4}$ and $\mathrm{H}_{2} \mathrm{O}$. Evaporation of the organic phase and FC (hexane/AcOEt gradient) gave the $p$ toluenosulfonil precursor of $\mathbf{1 0}$, which $(1.06 \mathrm{~g}, 3.9 \mathrm{mmol})$ was stirred with 7-hydroxy-2H-1-benzopyran-2-one (0.66 $\mathrm{g}, 4.1 \mathrm{mmol})$ and $\mathrm{NaH}(60 \%$ suspension in oil; $0.62 \mathrm{~g}, 12$ $\mathrm{mmol})$ in DMF $(35 \mathrm{~mL}$ ) for $24 \mathrm{~h}$. The mixture was then diluted with EtOAc and washed successively with $\mathrm{H}_{2} \mathrm{O}$ and $1 \mathrm{~mol} \mathrm{~L}^{-1}$ aqueous $\mathrm{NaOH}$. Evaporation of the organic phase and FC (hexane/EtOAc gradient) gave 10 (0.26 g, $26 \%$ ) as pale crystals; $\mathrm{mp} 67.9-68.9^{\circ} \mathrm{C}$ (EtOAc). ${ }^{1} \mathrm{H}$ NMR (300.067 MHz, $\left.\mathrm{CDCl}_{3}, \delta_{\text {TMS }} 0.00 \mathrm{ppm}\right) \delta 7.64\left(\mathrm{~d}, 1 \mathrm{H},{ }^{3} \mathrm{~J}\right.$ 9.3), $7.37\left(\mathrm{~d}, 1 \mathrm{H},{ }^{3} J 8.4\right), 6.84\left(\mathrm{dd}, 1 \mathrm{H},{ }^{3} J 8.4 ; 2.4\right), 6.80(\mathrm{~d}$, $\left.1 \mathrm{H},{ }^{3} \mathrm{~J} 2.4\right), 6.25\left(\mathrm{~d}, 1 \mathrm{H},{ }^{3} J 9.3\right), 4.27\left(\mathrm{t}, 2 \mathrm{H},{ }^{3} J 6.2\right), 4,11$ (t, $2 \mathrm{H},{ }^{3} J$ 6.2), 2.16 (qt, $2 \mathrm{H},{ }^{3} J 6.2$ ), 2.07 (s, $3 \mathrm{H}$ ); ${ }^{13} \mathrm{C}$ NMR $\left(75.45 \mathrm{MHz}, \mathrm{CDCl}_{3}, \delta_{\mathrm{CDCl}_{3}} 77.0 \mathrm{ppm}\right) \delta 170.7(\mathrm{C}), 161.7$ (C), $160.9(\mathrm{C}), 155.6(\mathrm{C}), 143.2(\mathrm{CH}), 128.6(\mathrm{CH}), 113.0$ $(\mathrm{CH}), 112.7(\mathrm{CH}), 112.5(\mathrm{C}), 101.2(\mathrm{CH}), 64.9\left(\mathrm{CH}_{2}\right), 60.9$ $\left(\mathrm{CH}_{2}\right), 28.4\left(\mathrm{CH}_{2}\right), 21.0\left(\mathrm{CH}_{3}\right)$;EIMS: $\mathrm{m} / z 262\left(\mathrm{M}^{+}, 100\right)$, 219 (7), 162 (29), 134 (72), 101 (83), 43 (89). The synthesis of compounds $\mathbf{3}$ and $\mathbf{1 1}$ were described elsewhere. ${ }^{13}$

\section{Microorganisms}

Yeasts and bacteria were cultured for 3 days, at room temperature, in $1.5 \mathrm{~mL}$ Eppendorf flasks respectively containing yeast-malt-agar medium $\left(3 \mathrm{~g} \mathrm{~L}^{-1}\right.$ yeast extract Merck, $3 \mathrm{~g} \mathrm{~L}^{-1}$ malt extract Merck, $5 \mathrm{~g} \mathrm{~L}^{-1}$ peptone Difco, $10 \mathrm{~g} \mathrm{~L}^{-1}$ glucose, $20 \mathrm{~g} \mathrm{~L}^{-1}$ agar Merck, $\mathrm{H}_{2} \mathrm{O}$ ) and Müller Hinton-agar medium (20 g L-1 Müller Hinton Merck, $20 \mathrm{~g}$ $\mathrm{L}^{-1}$ agar Merck, $\mathrm{H}_{2} \mathrm{O}$ ). Just prior to the tests the grown colonies were weighed into $1.5 \mathrm{~mL}$ Eppendorf flasks and suspended in aqueous buffer.

\section{Fluorescence measurements}

All buffers and solutions were prepared using MilliQdeionized $\mathrm{H}_{2} \mathrm{O}$. Microbial cells were diluted from $0.2 \mathrm{mg}$ $\mathrm{mL}^{-1}$ suspentions in $20 \mathrm{~mol} \mathrm{~L}^{-1}$ borate buffer ( $\mathrm{pH} \mathrm{8.8)}$. Substrates were diluted from $20 \mathrm{mmol} \mathrm{L}^{-1}$ stock solutions in $50 \%$ aqueous $\mathrm{MeCN}$. BSA was diluted from a $40 \mathrm{mg} . \mathrm{mL}^{-}$ ${ }^{1}$ stock solution in $20 \mathrm{mmol} \mathrm{L}^{-1}$ borate ( $\mathrm{pH} 8.8$ ). For the $\mathrm{EH}$ assay, $\mathrm{NaIO}_{4}$ was diluted from a $4.3 \mathrm{mg} \mathrm{mL}^{-1}$ aqueous solution. For BVMO assay, HLADH was diluted from a $10.6 \mathrm{mg} \mathrm{mL}^{-1}$ aqueous stock solution and $\mathrm{NAD}^{+}$was diluted from a $13 \mathrm{mg} \mathrm{mL}^{-1}$ aqueous stock solution. For screening $\mathrm{EH}$ activity, reactions were initiated by addition of $\mathrm{NaIO}_{4}$ to a solution containing substrate and BSA. For screening BVMO activity, reactions were initiated by addition of cells to a solution containing substrate, $\mathrm{HLADH}, \mathrm{NAD}^{+}$ and BSA. The $200 \mu \mathrm{L}$ assays were followed in individual wells of flat-bottom polypropylene 96 -well microtiter plates (Costar) with a Cytofluor-II fluorescence plate reader (Perseptive Biosystems, filters $\lambda_{\mathrm{ex}} 360 \pm 20 \mathrm{~nm}$, $\left.\lambda_{\mathrm{em}} 460 \pm 20 \mathrm{~nm}\right)$.

\section{Biocatalysis reactions}

Microbial cells weighed into $100 \mathrm{~mL}$ conic flasks (Costar) were suspended at $2 \mathrm{mg} \mathrm{mL}^{-1}$ in aqueous buffer (20 mmol L-1 borate $\mathrm{pH} 8.8$ or $20 \mathrm{mmo} \mathrm{L}^{-1}$ phosphate $\mathrm{pH} 6.5,25$ $\mathrm{mL})$. Then, a solution of ketone $3\left(50 \mu \mathrm{L}, 100 \mathrm{mg} \mathrm{mL}^{-1}\right.$ in $\mathrm{MeCN}$ ) was added to provide $0.2 \mathrm{mg} \mathrm{mL}^{-1}$ substrate solutions and the resulting mixtures were agitated on a plate stirrer at room temperature. To follow the reactions, $20 \mu \mathrm{L}$ aliquots were taken and analyzed by HPLC on a Chromolith Speed Rod column (RP-18e, $5.0 \times 10 \mathrm{~cm}$, Merck, flow $3.0 \mathrm{~mL} \mathrm{~min}^{-1}, 100 \%$ A to $100 \% \mathrm{~B}$ in $10 \mathrm{~min}$, A $=100 \% \mathrm{H}_{2} \mathrm{O}+0,1 \%$ TFA, $\mathrm{B}=60 \% \mathrm{MeCN}+40 \% \mathrm{H}_{2} \mathrm{O}+$ $0,1 \%$ TFA): $t_{\mathrm{R}} 4.1$ (7), 5.1 (11), 6.3 (9), 6.8 (10) $\mathrm{min}$. The integral of the peaks recorded by UV at $320 \mathrm{~nm}$ was used to calculate the conversion of released products.

\section{Conclusions}

An enzymatic HTS fluorogenic assay was smoothly adapted to a HTS protocol for EH of whole cells, and its 
application revealed 3 new microbial sources of $\mathrm{EH}(A$. tumefaciens, $P$. stipitis, and T. cutaneum). Considering the practical application of $\mathrm{EH}$ in organic synthesis, the utilization of the assay presented here for gathering selective biocatalysts is strongly recommended. On the other hand, the HTS fluorogenic assay based on Furstoss's procedure, ${ }^{14}$ used for present evaluation of monooxigenase, proved to be inadequate for cyclohexanone monooxigenase. Thus, a series of modifications are being evaluated. Notwithstanding this shortcoming the experiments revealed 2 sources of BVMO biocatalysts ( $G$. candidum and T. cutaneum). ${ }^{19}$

\section{Acknowledgements}

This work was supported by Fundação de Amparo à Pesquisa do Estado de São Paulo (FAPESP) and Conselho Nacional de Pesquisa e Desenvolvimento (CNPq).

\section{References}

1. Niessen, W.M.A.; J. Chromatogr. A 2003, 1000, 413.

2. Leroy, E.; Bensel, N.; Reymond, J.-L.; Bioorg. Med. Chem. 2003, 13, 2105.

3. Dean, K.E.S.; Klein, G.; Renaudet, O.; Reymond, J.-L.; Bioorg. Med. Chem. 2003, 13, 1653.

4. Wahler, D.; Badalassi, F.; Crotti, P.; Reymond, J.-L.; Angew. Chem. Int. Ed. 2001, 40, 4457.

5. Zocher, F.; Enzelberger, M.M.; Bornscheuer, U.T.; Hauer, B.; Schmid, R.D.; Anal. Chim. Acta 1999, 391, 345.

6. Cagnon, R.; Marsaioli, A.J.; Riatto, V.B.; Pilli, R.A.; Chemosphere 1999, 38, 2243.

7. Porto, A.L.M.; Cagnon, R.; Marsaioli, A.J.; Eguchi, S.Y.; Chemosphere 1999, 38, 2237.
8. Marsaioli, A.J.; de Conti, R.M.; Porto, A.L.M.; J. Mol. Cat. B. Enzym. 2001, 11, 233.

9. Ravot, G.; Wahler, D.; Favre-Bulle, O.; Cilia, V.; Lefevre, F.; Adv. Synth. Catal. 2003, 345, 691.

10. Mateo, C.; Archelas, A.; Furstoss, R.; Anal. Biochem. 2003, $314,135$.

11. Reetz, M.T.; Angew. Chem. Int. Ed. 2002, 41, 1335.

12. Klein, G.; Reymond, J.-L.; Helv. Chim. Acta. 1999, 82, 400.

13. Badalassi, F.; Wahler, D.; Klein, G.; Crotti, P.; Reymond, J.-L.; Angew. Chem. Int. Ed. 2000, 39, 4067.

14. Sleegers, A.; Simpson, H. D.; Alphand, V.; Furstoss, R.; The $5^{\text {th }}$ International Symposium on Biocatalysis and Biotransformation, Biotrans, 2001, Darmstadt, Germany.

15. Weijers, C.A.G.; Botes, A.L.; Van Dyk, M.S.; De Bont, J.A.M.; Tetrahedron: Asymmetry 1998, 9, 467.

16. Kronenburg, N.A.E.; Mutter, M.; Visser, H.; De Bont, J.A.M.; Weijers, C.A.G.M.; Biotechnol. Lett. 1999, 21, 519.

17. Porto, A.L.M.; PhD. Thesis, Universidade Estadual de Campinas, Brazil, 2002.

18. Kamerbeek, N.M.; Janssen, D.B.; van Berkel, W.J.H.; Fraaije, M.W.; Adv. Synth. Catal. 2003, 345, 667.

19. After submission of the present work we became aware of Furstoss's publication on the optimized method: Gutiérrez, M.C.; Sleegers, A.; Simpson, H. D.; Alphand, V.; Furstoss; Org. Biomol. Chem. 2003, 1, 3500 and the BVMO in G.candidum: Carballeira, J.D.; Alvarez, E.; Sinisterra. J.V.; J. Mol. Catalysis B, Enzymatic. 2004, 28, 25.

Received: September 22, 2003

Published on the web: November 12, 2004

FAPESP helped in meeting the publication costs of this article. 